\title{
Nano and Microscale Patterning on Soft Matters with Ion Beam Irradiation
}

\author{
Yeonuk Kim ${ }^{1}$, Jiachun Huang ${ }^{1}$, Ala Yasin Abuelfilat ${ }^{1}$, Jing $\mathrm{Fu}^{1}$ \\ ${ }^{1 .}$ Department of Mechanical and Aerospace Engineering, Monash University, Clayton, VIC 3800, \\ Australia
}

With high precision capability of Focused Ion Beam (FIB), we fine-tuned the surface topology and physical properties of thin film soft materials. The first example is hydrogel which is known for its biocompatibility, and both physical and chemical properties of hydrogels enable an environment preferred for cell growth and proliferation $[1,2]$. Surface properties at micro/nano scale also have a significant role at the interface of polymer/substrate to regulate cellular behaviors [3], and developing novel and effective surface modification approaches will be crucial for future tissue engineering applications. In this study, thin film hydrogel was coated on a silicon wafer followed by ion beam (keV $\mathrm{Ga}^{+}$) irradiation [4]. Sputtering yield of hydrogel as well as roughness and moduli were measured prior and after irradiation using Secondary Electron Microscope (SEM) and Atomic Force Microscopy (AFM).

Our results showed that nano and micro scale features can be patterned by FIB, with controllable feature size and physical attributes at the surface of hydrogel. The Young's modulus of irradiated hydrogel determined using AFM force spectroscopy was revealed to be dependent on ion fluence. Compared to the original Young's modulus value of $20 \mathrm{MPa}$, irradiation elevated the value to $250 \mathrm{MPa}$ and $350 \mathrm{MPa}$ at $1 \mathrm{pC} \mu \mathrm{m}^{-2}$ and $100 \mathrm{pC} \mu \mathrm{m}^{-2}$ respectively. Cell culture studies confirmed that the irradiated hydrogel samples were biocompatible, with stable nanoscale patterns under physiological conditions [4]. This patterning approach can also be extended to other soft samples such as tumour tissues (Figure 2a). Monte Carlo simulation was performed first to investigate the ion-tissue interactions, based on the chemical compositions from database and EDX mapping (Figure 2b), followed by deploying FIB as a digitally controlled slicing tool. Site specific probing of thin section tumour tissue could be achieved with AFM in various imaging modes (Figure 2c), with minimal effects from the microtome sectioning.

\section{References:}

[1] A. Gaston, et al, "Nanopatterned UV curable hydrogels for biomedical applications" Microelectronic engineering, vol. 87 (2010), pp. 1057-1061.

[2] A. Al-Abboodi, et al, "Three-Dimensional nanocharacterization of porous hydrogel with ion and electron beams" Biotechnology and bioengineering, vol. 110, no. 1 (2013), pp.318-326.

[3] F. Boccafoschi, et al, "Study of cellular adhesion by means of micropillar surface topologies" Advanced materials research, vol. 409 (2012) pp. 105-110.

[4] Y. Kim, et al, "Tuning the surface properties of hydrogel at the nanoscale with focused ion irradiation" Soft Matter, vol. 10 (2014), pp. 8848-8456.

[5] Funding for this research was partly provided through Australia Research Council Discovery Project Grant (DP120102570 and DP120100583) and Interdisciplinary Research Seed Fund of Monash University. This work was performed in part at the Melbourne Centre for Nanofabrication (MCN) in the Victorian Node of the Australian National Fabrication Facility (ANFF). The author would like to thank the staff from MCN and Anatomical Pathology, Alfred Hospital for various supports. 
a
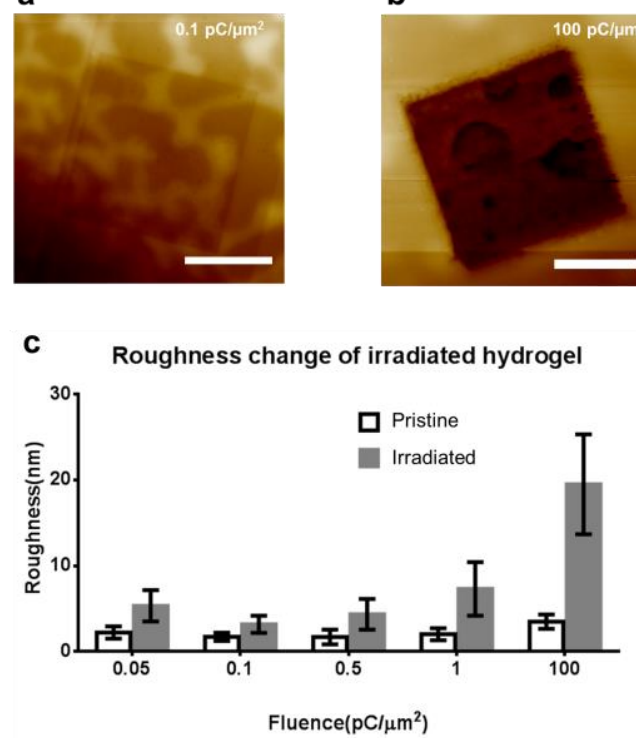

b
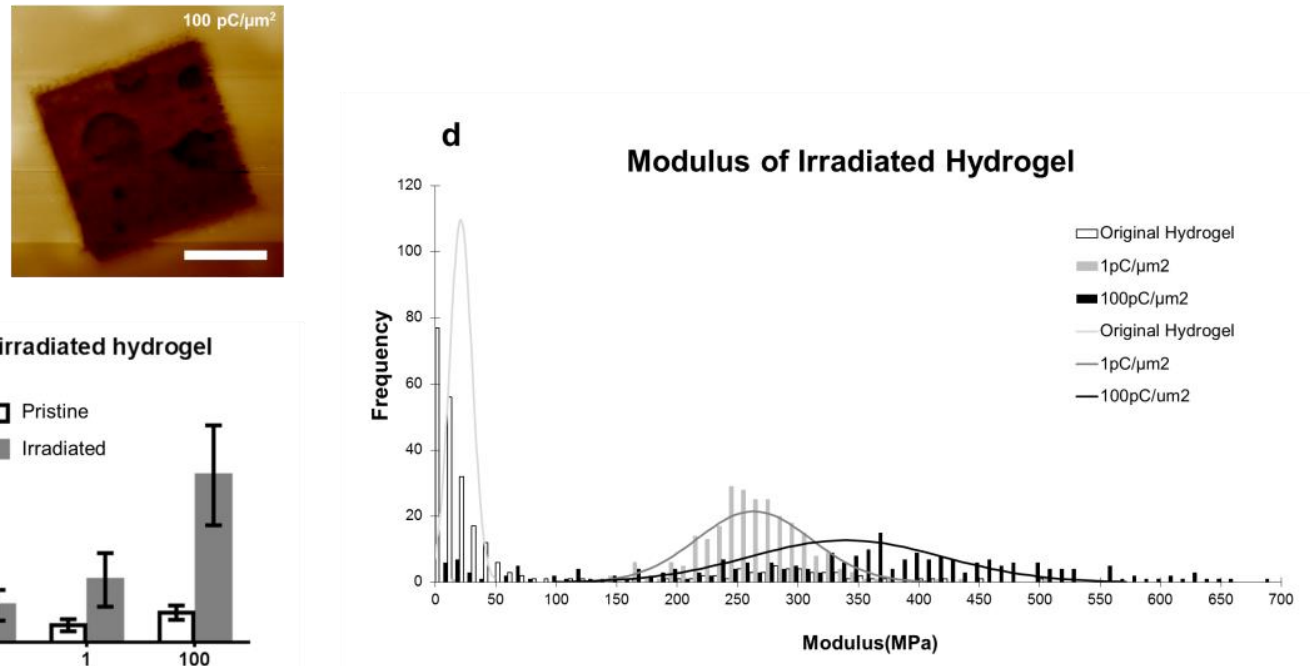

Figure 1. AFM images (top view) of surface morphology after ion milling $\left(\mathrm{Ga}^{+}, 30 \mathrm{kV}\right)$ with ion fluence (a) $0.1 \mathrm{pC} / \mu \mathrm{m}^{2}$ and (b) $100 \mathrm{pC} / \mu \mathrm{m}^{2}$. (c) The corresponding numerical values of surface roughness of pristine hydrogel and after ion irradiation with increasing ion fluence. (Scale bar: $5 \mu \mathrm{m})(\mathrm{d})$ Modulus of hydrogel before and after the ion beam irradiation.
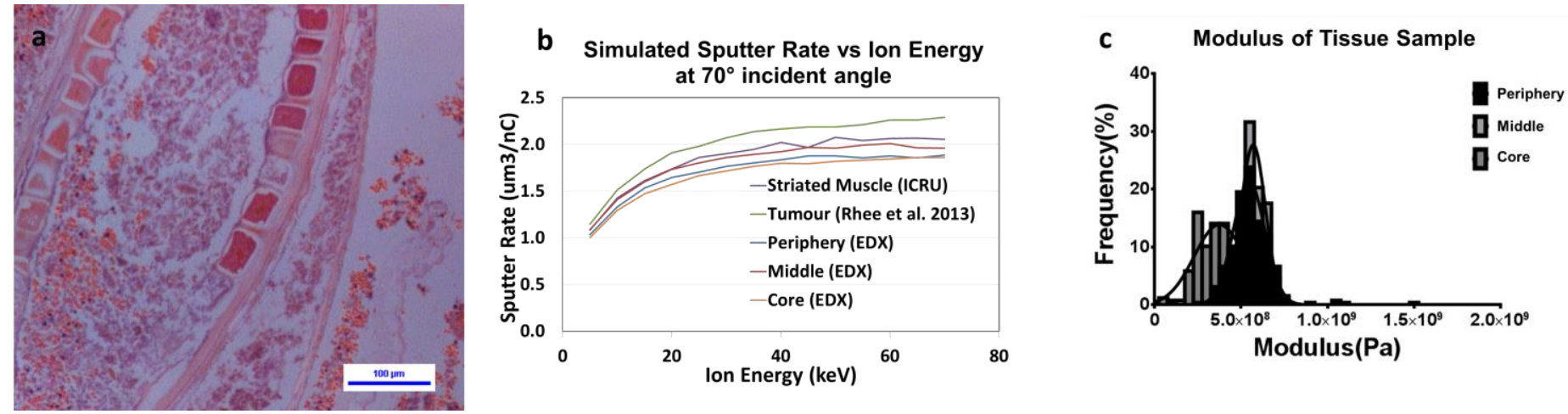

Figure 2. (a) Optical image of the multilayer structures of tumour tissue, and (b) Monte Carlo simulation of ion-tissue interactions with target properties based on database and EDX readings. (c) Young's modulus obtained in different layers of the tumour tissue sample by AFM force spectroscopy. 